\title{
Importance of a registered and structured protocol when conducting systematic reviews: comments about nebulized antibiotics for ventilator-associated pneumonia
}

\author{
Fernando G. Zampieri ${ }^{1,2,3}$, Antonio P. Nassar Jr ${ }^{1,2,4}$, Dimitri Gusmao-Flores ${ }^{1,5}$, Leandro U. Taniguchi ${ }^{2,6}$, Antoni Torres ${ }^{7}$ \\ and Otavio T. Ranzani $i^{1,7,8, *^{*}}$
}

See related Letter by Gu, http://www.ccforum.com/content/19/1/236, and related research by Zampieri et al., http://ccforum.com/content/19/1/150

We appreciate Gu's [1] interest in our study. We apologize and agree with his comment about attributing units to standardized mean difference (SMD). Nevertheless, similar to the SMD, results in mean difference (control - nebulized) were unaffected by nebulized antibiotics (2.67 days, $95 \%$ confidence interval (CI) $-2.89,8.23$ for ICU length of stay (LOS); and 0.70 days, $95 \% \mathrm{CI}-3.40,4.80$ for mechanical ventilation). However, we strongly disagree with other points raised by the letter.

First, the study protocol was defined a priori [2]. We disagree that combining observational studies with intervention studies is reserved only for safety evaluation. This topic has been discussed in the literature and combining both types of studies was adequate for our aim [3]. Furthermore, we presented the main results separating interventional studies from observational studies, thereby allowing the reader to interpret both analyses independently.
Second, both of the studies cited as "case-control studies" [1] received this denomination in their title and abstract. However, by reading their methods it becomes clear that they are actually matched cohort studies $[4,5]$. Indeed, they matched exposed patients ("nebulized group") to unexposed patients ("no-nebulized group"). A case-control design starts with the outcome (case $=$ "clinical success") and matches them with controls ("clinical failures"). Therefore, our measure of effect was correct [5]. For exploration, we report the analysis for clinical cure using the odds ratio (OR) (Fig. 1). The results are unchanged.

Third, Kalin's study was included because it fulfilled our inclusion/exclusion criteria [2]. Gu's suggestion to exclude this study based solely on its effects in heterogeneity could be considered selective reporting [1].

Our study provided data for further trials aiming to evaluate the effect of nebulized antibiotics in ventilatorassociated pneumonia (VAP) [2].

* Correspondence: otavioranzani@yahoo.combr

${ }^{1}$ Cooperative Network for Research-AMIB-Net, Associação de Medicina Intensiva Brasileira, Rua Arminda, 93, 7 andar, São Paulo 04545-100, Brazil ${ }^{7}$ Department of Pulmonology, Hospital Clinic of Barcelona, Institut D'investigacions August Pi I Sunyer (IDIBAPS), University of Barcelona, Ciber de Enfermedades Respiratorias (CIBERES), Carrer Villarroel, 170, Barcelona 08036, Spain

Full list of author information is available at the end of the article 


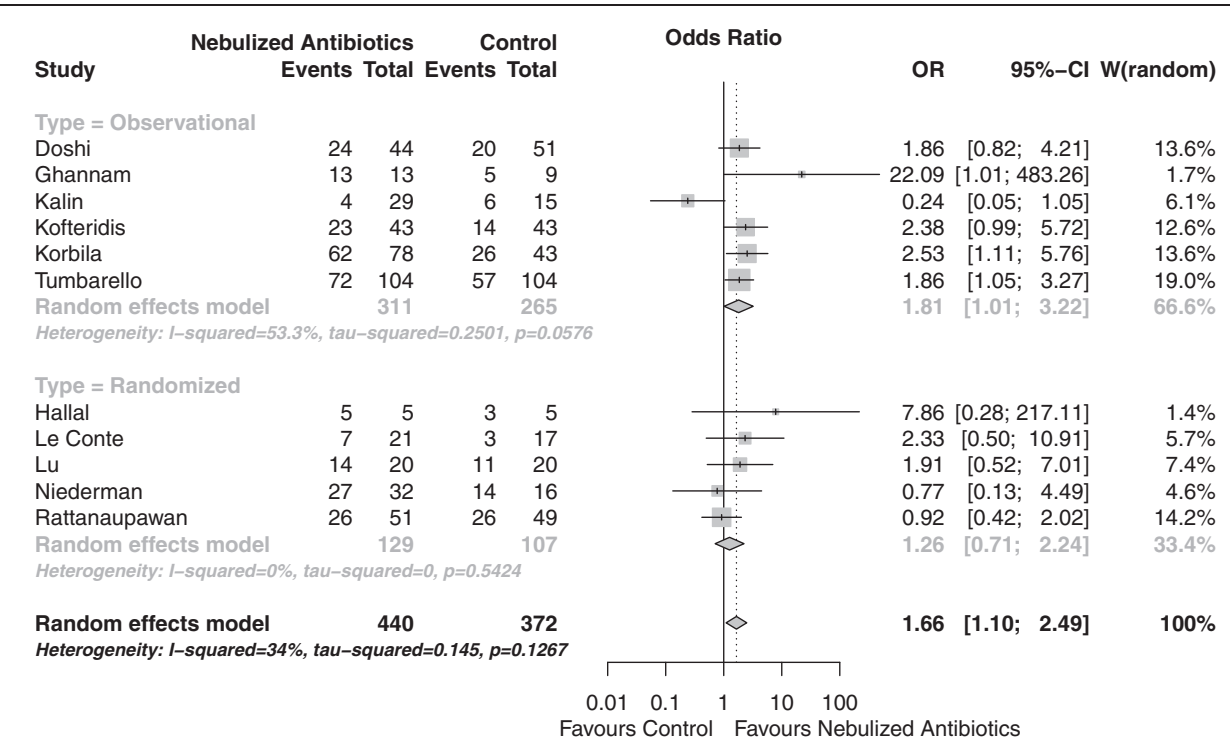

Fig. 1 Forest plot for clinical cure using odds ratios (OR). $P$ for overall effect $=0.015$. Cl confidence interval

\section{Abbreviations}

$\mathrm{Cl}$ : Confidence interval; LOS: Length of stay; OR: Odds ratio;

SMD: Standardized mean difference; VAP: Ventilator-associated pneumonia.

\section{Competing interests}

The authors declare that they have no competing interests.

\section{Author contributions}

FGZ conceived the study, participated in data acquisition, data analysis, and interpretation, and helped to revise the manuscript for important intellectual content. APN participated in conception of the study, data acquisition, data analysis, and interpretation, and helped to revise the manuscript for important intellectual content. DG-F participated in conception of the study and interpretation, and helped to revise the manuscript for important intellectual content. LUT participated in conception and design of the study and interpretation, and helped to revise the manuscript for important intellectual content. AT participated in conception and design of the study and interpretation, and helped to revise the manuscript for important intellectual content. OTR conceived the design of the study, participated in data acquisition and interpretation, and wrote the draft of the manuscript. All authors read and approved the final manuscript.

\section{Author details}

'Cooperative Network for Research—AMIB-Net, Associação de Medicina Intensiva Brasileira, Rua Arminda, 93, 7 andar, São Paulo 04545-100, Brazil. ${ }^{2}$ Emergency Medicine Discipline, Faculty of Medicine, University of São Paulo, Rua Dr. Enéas de Carvalho Aguiar, 255, 5th floor, room 5023, São Paulo 05403-010, Brazil. ${ }^{3}$ Intensive Care Unit, Hospital Alemão Oswaldo Cruz, Rua João Julião, 331, São Paulo 01323-903, Brazil. ${ }^{4}$ Adult Intensive Care Unit, A.C. Camargo Cancer Center, Rua Professor Antônio Prudente, 211, São Paulo 01509-010, Brazil. ${ }^{5}$ Intensive Care Unit, University Hospital Prof. Edgar Santos, Universidade Federal da Bahia, Rua Augusto Viana, Salvador 40110-910, Brazil. ${ }^{6}$ Research and Education Institute (IEP), Hospital Sirio-Libanes, Rua Prof. Daher Cutait, 69, São Paulo 01308-060, Brazil. Department of Pulmonology, Hospital Clinic of Barcelona, Institut D'investigacions August Pi I Sunyer (IDIBAPS), University of Barcelona, Ciber de Enfermedades Respiratorias (CIBERES), Carrer Villarroel, 170, Barcelona 08036, Spain. ${ }^{8}$ Amil Critical Care Group, Hospital Paulistano, Rua Martiniano de Carvalho, 741, São Paulo 01321-001, Brazil. ${ }^{9}$ Respiratory Intensive Care Unit, Pulmonary Division, Heart Institute, Hospital das Clínicas, University of São Paulo, Av. Dr. Arnaldo, 455 Laboratório de Pneumologia, $2^{\circ}$ andar, sala 2144, Cerqueira César, 01246903 Sao Paulo, Brazil.

\section{Published online: 20 August 2015}

\section{References}

1. Gu WJ. Nebulized antibiotics for ventilator-associated pneumonia: misleading analysis and interpretation of the data. Crit Care. 2015;19:236.

2. Zampieri FG, Nassar AP, Gusmao-Flores D, Taniguchi LU, Torres A, Ranzani OT. Nebulized antibiotics for ventilator-associated pneumonia: a systematic review and meta-analysis. Crit Care. 2015;19:150.

3. Shrier I, Boivin JF, Steele RJ, Platt RW, Furlan A, Kakuma R, et al. Should meta-analyses of interventions include observational studies in addition to randomized controlled trials? A critical examination of underlying principles. Am J Epidemiol. 2007;166:1203-9.

4. Cummings P, McKnight B, Greenland S. Matched cohort methods for injury research. Epidemiol Rev. 2003;25:43-50.

5. Rothman KJ, Greenland S, Lash TL. Modern epidemiology. 3rd ed. Philadelphia, PA: Lippincott Williams \& Wilkins; 2008. 